\title{
llizarov bone transport in large knee joint defect (case report)
}

\begin{abstract}
The large knee joint defects (LKJD) are one of the indications to amputation and prosthesis. Alternative to it is reconstructive surgery based on Ilizarov method. We present the case of a female patient with LKJD $16 \mathrm{~cm}$ and shortening of the right lower extremity $12 \mathrm{~cm}$, hypertrophic distraction regenerate of the right lower limb $6 \mathrm{~cm}$ and chronic osteomyelitis of the right femur. The whole period of treatment was 67 months (5.5 years). Period of osteosynthesis (period of distraction and fixation) was 43 months ( 3.5 years). The complications that we faced during the treatment had no influence on good final anatomical and functional results.
\end{abstract}

Keywords: knee joint, ilizarov method, oncology, osteomyelitis, surgery
Volume 2 Issue I - 2018

\section{LN Solomin,, ${ }^{1,2}$ KL Korchagin,' EO Tyulkin,' EA Shchepkina ${ }^{1,3}$ \\ 'Russian Vreden Scientific Research Institute of Traumatology and Orthopaedics of the RF Ministry of Health, Russia \\ ${ }^{2}$ St Petersburg State University, Medical Faculty, Russia \\ ${ }^{3}$ Pavlov First Saint Petersburg State Medical Academy, Russia}

Correspondence: LN Solomin, Russian Vreden Scientific Research Institute of Traumatology and Orthopaedics of the RF Ministry of Health, Medical Faculty, St Petersburg State University, Email solomin.leonid@gmail.com

Received: November 01, 2017 | Published: February 02, 2018

\section{Introduction}

The large knee joint defects (LKJD) leads to persistent dysfunction of the lower limbs and disability. ${ }^{1,2}$ The most common reason of extended defects of the bones forming knee joint is a radical surgery for osteomyelitis, failed revision arthroplasty for oncology and Endoprosthesis replacement. ${ }^{3-5}$ In case of impossibility of an operation for the next revision endoprosthetics of knee joint, the alternative to amputation and exoprosthetics is the operation of arthrodesis of the knee joint. ${ }^{2,6-8}$ However, the presence of LKJD makes it impossible for acute shortening due to the invagination of soft tissue, vascular and neurological disorders. Therefore, for this kind of patients the reconstructive surgery based on of Ilizarov method is indicated. .,2, $^{2}$

\section{Clinical observation}

Female, 55y.o. Giant cell tumor of bone of the distal third of the right femur was diagnosed in 1985. Resection with subsequent arthroplasty was performed. In 1993 and 2002 revision arthroplasty were done. In 2003 a deep infection developed. On June 05, 2003 debridement and removal of an Endoprosthesis were performed. As a result, a large knee joint defect $30 \mathrm{~cm}$ was formed.

In 2004 following manipulations were performed: plastic defect with vascularized fibular graft, corticotomy of the tibia, attempt of bone defect replacement according to Ilizarov. As a result, the consolidation occurred only at the site of contact between the graft and the tibia; a hypoplastic regenerate in the middle third of the lower leg was formed. In 2006 she was admitted to the Russian Scientific Research Institute of Traumatology and Orthopedics named after R.R. Vreden with a diagnosis: $16 \mathrm{~cm}$ of defect of the bones forming the right knee joint, $12 \mathrm{~cm}$ of the right lower limb shortening, $6 \mathrm{~cm}$ of hypertrophic distraction regenerate of the right tibia, chronic osteomyelitis of the right femur and lower leg, remission phase (Figure 1).

The patient refused amputation, which she has been repeatedly proposed. The first stage of treatment included the operation performed on 25 January 2006: the combined (hybrid) external fixation device (EFD) was imposed, corticotomy with osteoclasia of the middle third of the tibia, subsequent distraction to replace the defect and compression at the levels of hypertrophic regenerate and defect were performed (Figure 2).

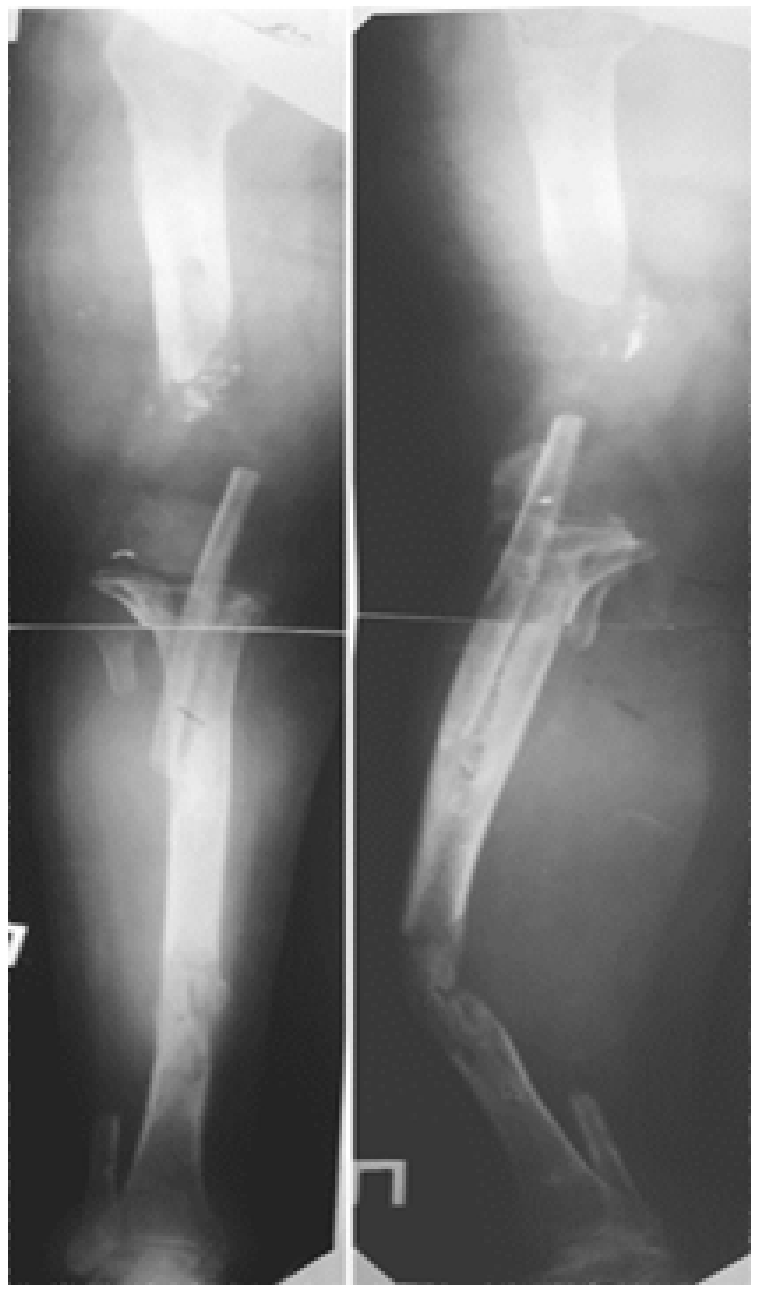

Figure I Radiographs before treatment. 


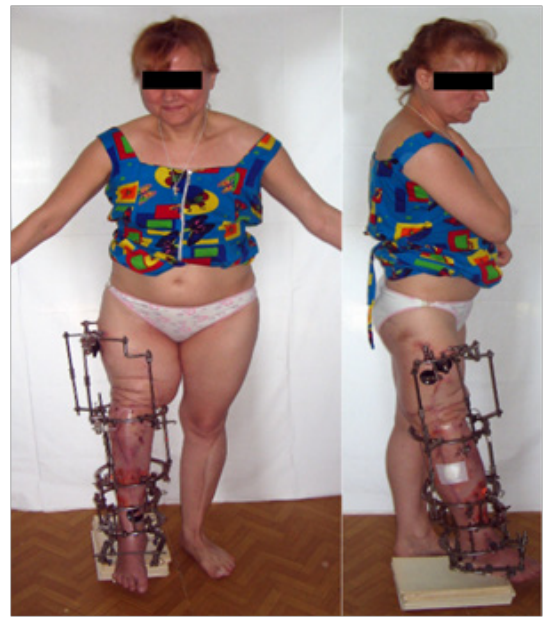

A

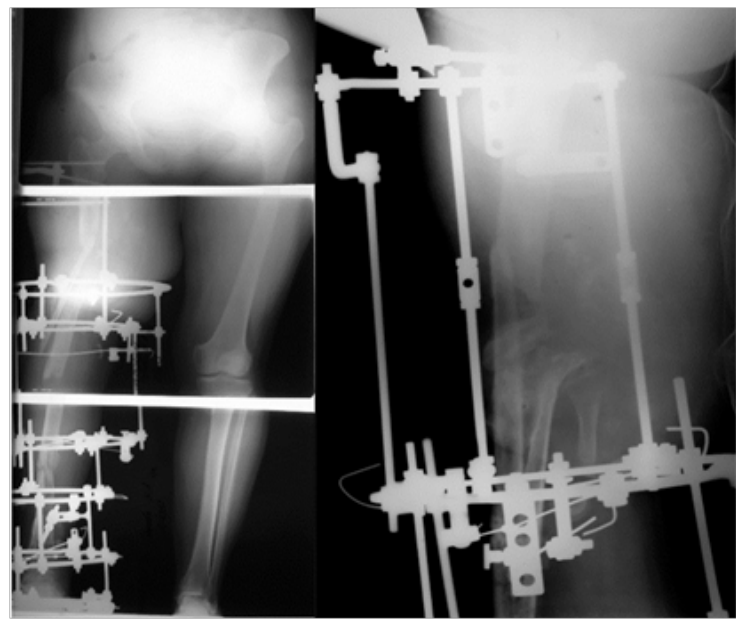

B

Figure 2A \& B Photos and radiographs of the patient after application of combined (hybrid) external fixation device (EFD), cortycotomy with osteoclasia of the middle third of the tibia, subsequent distraction to replace the defect and compression at the levels of the hypertrophic regenerate and the defect.

From 6 weeks beginning of treatment cortycotomy with osteoclasia of the femur and subsequent distraction to replace the defect of the bones forming the knee joint were performed. For 120 days/17 weeks of distraction received distraction regenerate at the femur with a length of $8 \mathrm{~cm}$, on the lower leg $13 \mathrm{~cm}$ length (Figure 3 ).
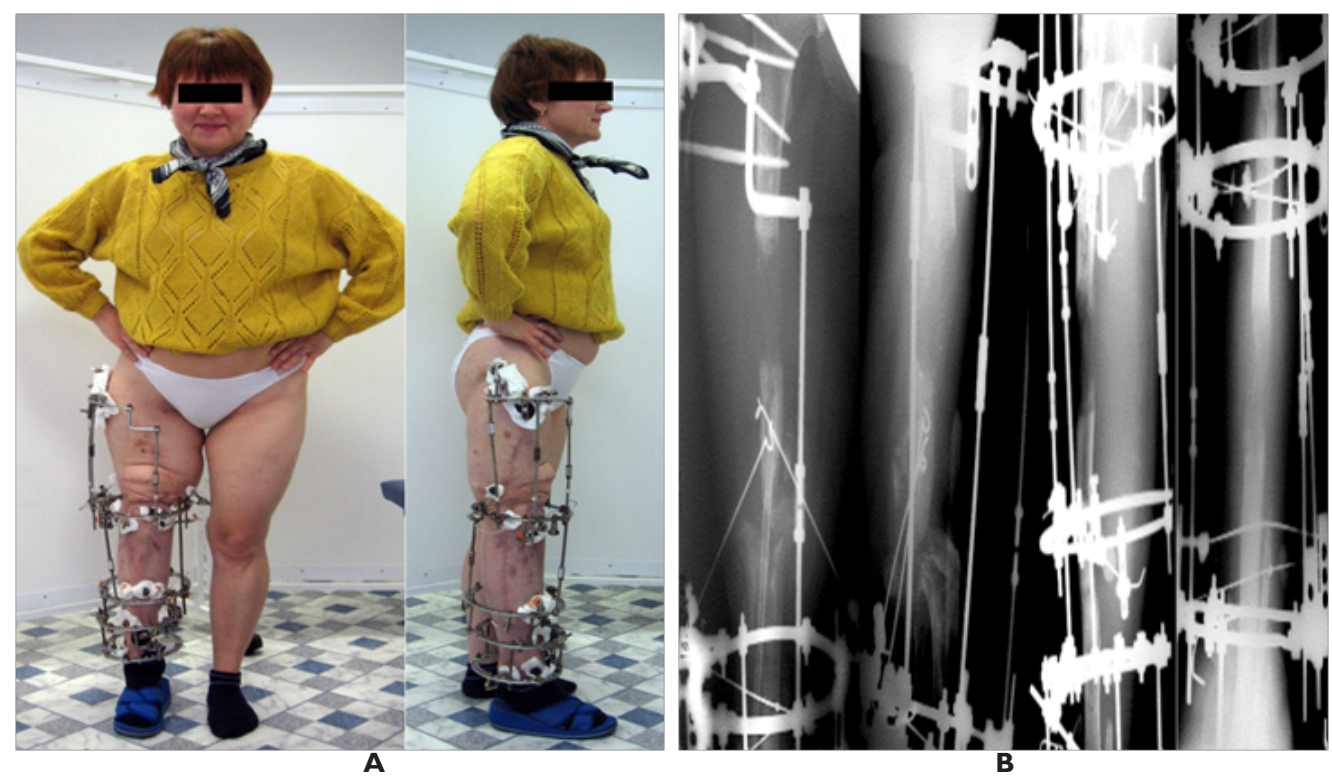

Figure 3A \& B Photos and radiographs of the patient after cortycotomy with osteoclasia of the femur and subsequent distraction to replace the defect of the bones forming the knee joint.

The second stage of treatment included: on 26 March 2007 [from 60 weeks beginning of treatment] an open adaptation at the junction of bone fragments of the femur and tibia was performed. For the formed equinus foot position of the right foot, Achilles tendon was lengthened, and hinges transosseous apparatus was applied (Figure 4).

The period of osteosynthesis was 30 months/132 weeks (the period of distraction of 12 months $/ 53$ weeks+fixation period of 18 months/79 weeks) 10.09.08. EFD was dismantled (Figure 5). The residual shortening of the right lower limb at that time was up to $8 \mathrm{~cm}$. The third stage of treatment included: On 05 October 2010 [From 248 weeks the beginning of treatment/62 months] the imposition of EFD and cortycotomy with osteoclasia of the right femur was performed.
The distraction was carried out during 110 days/16 weeks. As a result, regenerate with a length of $8 \mathrm{~cm}$ was formed. At the end of distraction, the correction of the mechanical axis of the lower limb with Ortho-SUV device was carried out. ${ }^{10}$ The period of osteosynthesis of this stage was 13 months $/ 52$ weeks: the distraction period was 3.5 months $/ 14$ weeks, and the fixation period was 9.5 months $/ 38$ weeks (Figure 6). Thus, the total duration of treatment was 67 months. (5.5 years). The total period of osteosynthesis (the distraction period+the fixation period) was 43 months. (3.5 years). During the treatment, due to the long period of osteosynthesis in the EFD, soft tissue inflammation repeatedly occurred in the area of the transosseous element outlet that was stopped by local application of antibiotics 
and bandages (category 1 complication according to Caton). ${ }^{11,12}$ The remounting of EFD was required twice due to the instability of

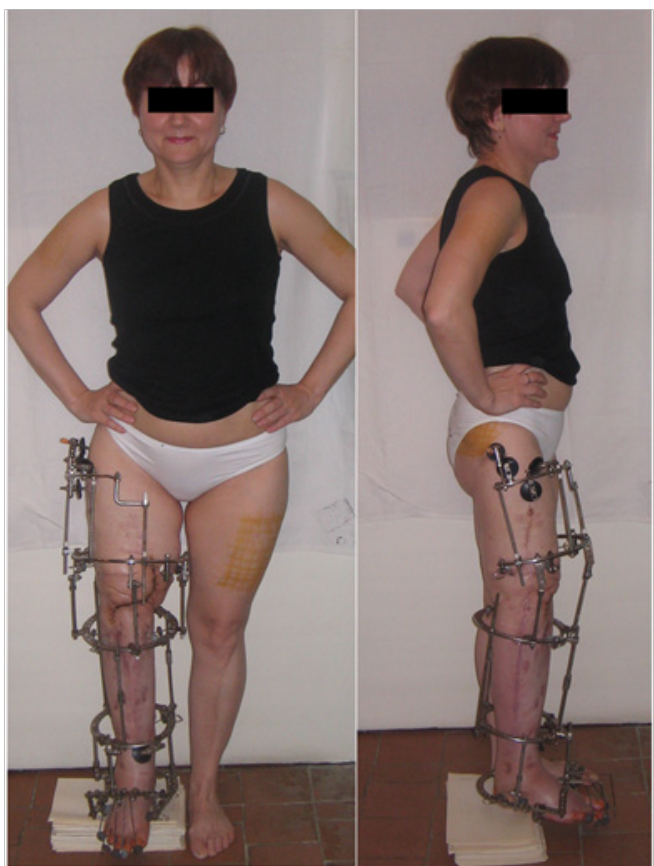

A transosseous elements (category 2 complications, according to Caton) that did not affect the treatment result.

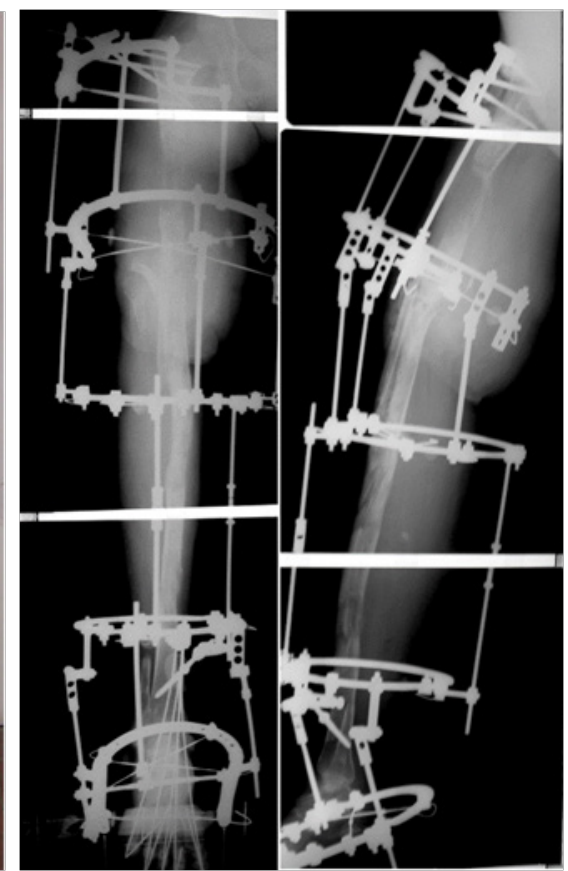

Figure 4A \& B Photos and radiographs of the patient after achilles tendon lengthening and application of hinge transosseous apparatus to eliminate equinoxes foot position.

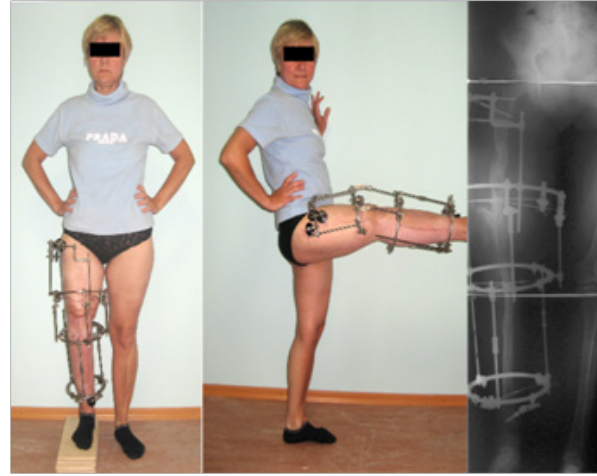

A

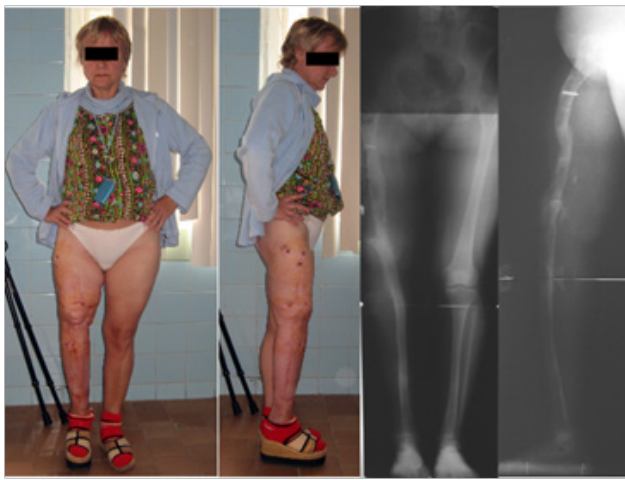

B

Figure 5 Photos and radiographs of the patient in the (A) Fxation phase and (B) After dismantling of EFD.

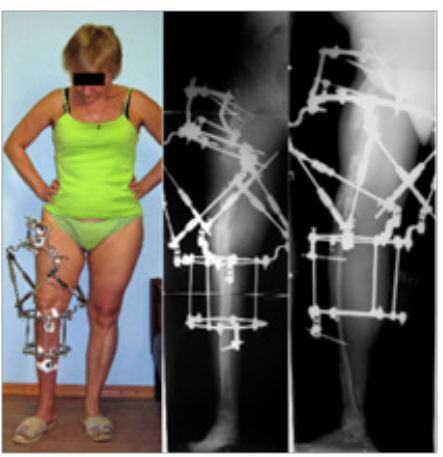

A

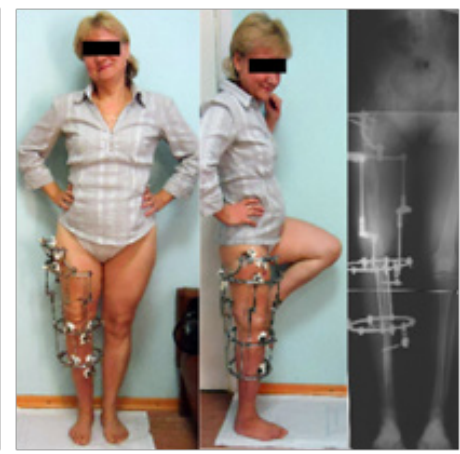

B

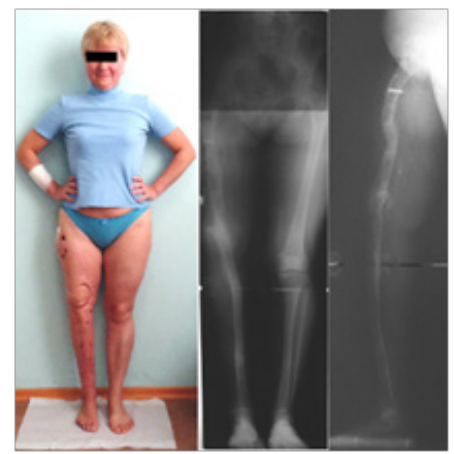

C

Figure 6 Photos and radiographs of the patient $(\mathbf{A})$ In the elimination of residual shortening and correction of the mechanical axis of the lower limb, (B) In the fixation phase, (C) After the dismantling of EFD. 
The long-term result was estimated in November 2016, 5 years after the completion of the third stage of treatment (Figure 7). The patient walks without additional means of support and uses a walking cane only for a long distance walking. The right lower limb is completely supportable, no disturbed circulations and innervations are observed. The lengths of the lower limbs are equal, the mechanical axes of the right lower limb are correct. The patient drives a car with hand control. The patient is married, brings up a child.

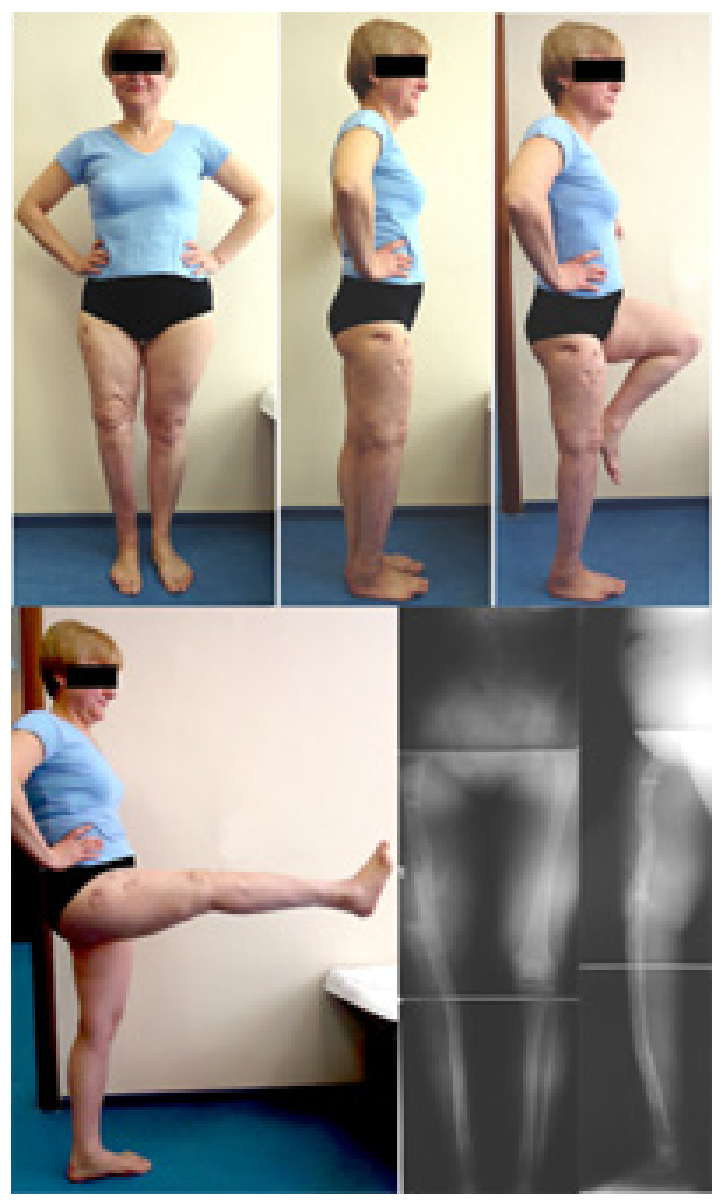

Figure 7 Photos and radiographs of the patient 5 years after the completion of treatment.

\section{Discussion}

The frequency of infectious complications after total endoprosthetics of the knee joint, according to different authors, varies from $0.57 \%$ to $15 \% .{ }^{9}$ Revision surgery, the installation of the hinge model of the Endoprosthesis - all this increases the risk of infection. ${ }^{13}$ Therefore, complications of endoprosthetics are currently the most frequent reasons for performing arthrodesis of the knee joint, when the presence of chronic osteomyelitis is a contraindication to performing revision endoprosthetics. ${ }^{8}$ The shortening of the operated limb after arthrodesis of the knee joint, after joint replacement according to different authors ranges from 1.5 to $6.4 \mathrm{~cm} .{ }^{8,14}$ In case of the large bone defect forming the knee joint after removal of cancer endoprosthesis staged reconstructive interventions are recommended..$^{8,13,15,16}$ There is strong evidence that arthrodesis of the knee joint is preferable to amputation. ${ }^{8,15}$ Amputation after total endoprosthetics of the knee joint significantly reduces the quality of life, due to the impaired ability to move. ${ }^{17,18}$
However, there are few cases in the literature describing successful treatment of patients with LKJD after endoprosthetics with oncological endoprosthesis. We found only 3 publications describing the cases of substitution of extensive LKJD after a failed endoprosthesis replacement with oncological prostheses. ${ }^{15,16}$ Tokizaki cites 3 cases of treatment of patients with defects after removal of oncological prostheses with a defect value of $22-33 \mathrm{~cm}$, the average fixation time was 19.4 months (14.7-24.2). Kinik (2009) describes 3 cases, one of which is post-traumatic defects of 11 to $31 \mathrm{~cm}$, the mean fixation time was 10.1 months $(7.5-15)$, the follow-up period was 33.6 months (25-39 months). Hatzokos describes 2 cases of substitution of extensive LKJD from which in one case the oncological prosthesis was useded after a severe fracture of the proximal tibia Schatzrer VI. The size of defects was 19 and $25 \mathrm{~cm}$ with a fixation period of 27 and 34.7 months, respectively. Other publications ${ }^{18-20}$ describe cases of replacement of posttraumatic defects. All the authors describe complications, characteristic for external fixation - pin-tract infection ${ }^{11,12}$ and, in a number of cases, secondary foot deformity after tibia lengthening. ${ }^{13,15,18,19}$ The results of the SF-36 scale indicated that treatment significantly improved both the physical and psychological quality of life of patients. ${ }^{13,18}$

The main advantage of external fixation authors considers the simultaneous removal of limb length discrepancy and weight bearing restoration. ${ }^{20}$ The arthrodesis of the knee joint in the treatment of LKJD reduces the risk of repeated deep infection. Among the shortage of the method is an extremely long period of fixation. However, if the recommendations are observed, this does not worsen the result obtained..$^{13}$ The result obtained by us on the terms of treatment, the period of fixation is not inferior to the literature data. ${ }^{21}$

\section{Conclusion}

The treatment of this patient with a large bone defect forming the knee joint was multi-staged, long-term and labor-intensive. However, this treatment was alternative to amputation and exoprosthetics, which the patient categorically refused. The patient is completely satisfied with the obtained results and confirms the correct choice of the therapeutic approach.

\section{Acknowledgements}

None.

\section{Conflict of interest}

The author declares no conflict of interest.

\section{References}

1. Klyushin NM, Shlyakhov VI, Chakushin BE, et al. Transosseous osteosynthesis in the treatment of patients with chronic osteomyelitis after endoprosthetics of large joints. Genius of Orthopedics. 2010;2:3743.

2. Klyushin NM, Ababkov Yu V, Burnashov SI, et al. The result of arthroplasty of the knee joint after septic arthritis by the method of bilocal compression-distraction osteosynthesis according to Ilizarov with bone patella plasty (case from practice). Genius of Orthopedics. 2014;4:89-92.

3. Bovkis G Yu, Kulyaba TA, Kornilov NN. Compensation of defects of metaepiphysis of the femoral and tibia bones with revision knee joint endoprosthetics - methods and results of their use. Traumatology and orthopedics of Russia. 2016;22(2):101-113. 
4. Dedkov AG. Arthrodetic surgical interventions in orthopedic oncology Orthopedics, traumatology and prosthetics. 2009;4:40-45.

5. Kulyaba TA, Kornilov NN, Selin AV, et al. Methods of compensation of bone defects with revision knee joint endoprosthesis. Traumatology and Orthopedics of Russia. 2011;3:5-12.

6. Prokhorenko VM, Zlobin AV, Mamedov AA, et al. Treatment of paraprosthetic infection of the knee joint. Modern problems of science and education. 2015:6.

7. Balci HI, Saglam Y, Pehlivanoglu T, et al. Knee arthrodesis in persistently infected total knee arthroplasty. The journal of knee surgery. 2016;29(7):580-588.

8. Conway JD, Mont MA, Bezwada HP. Arthrodesis of the knee. $J$ Bone Joint Surg Am. 2004;86-A(4):835-848.

9. Kuchinad R, Fourman MS, Fragomen AT, et al. Knee arthrodesis as limb salvage for complex failyres of total knee arthroplasty. J Arthroplasty. 2014;29(11):2150-2155.

10. Solomin LN, Shchepkina EA, Vilensky VA, et al. Correction of deformities of the femur by Ilizarov and computer-based Ortho-SUV apparatus. Traumatology and Orthopedics of Russia. 2011;32-39.

11. Lascombes P, Popkov D, Huber H, et al. Classification of complications after progressive long bone lengthening: Proposal for a new classification. Orthop Traumatol Surg Res. 2012;98(6):629-637.

12. Paley D. Problems, obstacles and complications of limb lengthening by the Ilizarov technique. Clin Orthop Relat Res. 1990;250:81-104.

13. Hatzokos I, Stavridis SI, Iosifidou E, et al. Distraction osteogenesis as a salvage method in infected knee megaprostheses. Knee. 2010;18(6):470 473.
14. Salem KH, Keppler P, Kinzl L, et al. Hybrid external fixation for arthrodesis in knee sepsis. Clin Orthop Relat Res. 2004;451:113-120.

15. Kinik H. Knee Arthrodesis with Ilizarov's Bone Transport Method for Large Infected Periarticular Defects: A Report of Three Cases. J Trauma. 2009;67(6):E213-E219.

16. Tokizaki T, Abe S, Tateishi A, et al. Distraction osteogenesis for knee arthrodesis in infected tumor prostheses. Clin Orthop Relat Res. 2004;(424):166-172.

17. Christie MJ, DeBoer DK, McQueen DA, et al. Salvage procedures for failed total knee arthroplasty. J Bone Joint Surg Am. 2003;85-A(Suppl 1):58-62.

18. Rozbruch SR, Ilizarov S, Blyakher A. Knee arthrodesis with simultaneous lengthening using the Ilizarov method. J Orthop Trauma. 2005;19(3):171-179.

19. Barwick TW, Montgomery RJ. Knee arthrodesis with lengthening: Experience of using Ilizarov techniques to salvage large asymmetric defects following infected peri-articular fractures. Injury. 2013;44(8):1043-1048.

20. Manzotti A, Pullen C, Guerreschi F, et al. Knee arthrodesis and limb lengthening in the treatment of infected total knee arthroplasty:case report. J Trauma. 2002;52(2):359-363.

21. Oostenbroek HJ, van Roermund PM. Arthrodesis of the knee after an infected arthroplasty using the Ilizarov method. J Bone Joint Surg Br. 2001;83(1):50-54. 